\title{
A study of the unsteady aerodynamics of a wing at high angles of attack using decambering to model separated flow
}

\author{
B ANTONY SAMUEL*(D) and RINKU MUKHERJEE \\ Indian Institute of Technology, Madras, Chennai 600036, India \\ e-mail: antonysamuelb@gmail.com; rinku@iitm.ac.in
}

MS received 16 May 2017; revised 9 December 2017; accepted 18 January 2018; published online 27 June 2018

\begin{abstract}
An Unsteady Vortex Lattice Method is developed and validated. It is coupled with a decambering methodology to account for viscous effects on the aerodynamic coefficients. Two additional methodologies to select a unique solution when multiple solutions arise have been proposed. The transient nature of the aerodynamic loads of a suddenly moving wing at different angles of attack is examined. Sudden jumps are observed in the $C_{L}(t)$ at post-stall angles of attack. The jumps are followed by the presence of asymmetric solutions, which then decline with time and a change in the solution state. Higher angles of attack see an increasing number of jumps.
\end{abstract}

Keywords. Wing; aerodynamics; unsteady; post-stall; separated flow; high angle of attack; decambering.

\section{Introduction}

Post-stall flow phenomenon is inherently unsteady and several parameters contribute to its unsteadiness ranging from the angle of attack, shape and motion of the wing to the turbulence in the freestream. Prediction of even steady flow over wings at post-stall angles of attack $(\alpha)$ is in itself resource intensive and hence methods such as the strip theory and predictions based on neural networks have been developed for real time flow prediction.

Strip theory assumes each section of a wing to act like an aerofoil. The relationship between the $C_{L}$ and $\alpha$ at each section is governed by the $C_{L}-\alpha$ curve provided as input. Such a method was first implemented by Tani [1] in 1934. Schairer [2] developed a similar method in 1939 and reported the presence of asymmetry and existence of multiple solutions over certain ranges of $\alpha$. The author further commented that additional information was required to choose between the available multiple solutions. Piszkin and Levinsky [3] (1976) used an Unsteady Vortex Lattice Method (UVLM) with a single vortex ring at each section of the wing. The authors studied variation of solutions with different parameters and the effect of the negative $C_{L}-\alpha$ slopes. Variations of the strip theory were later on implemented by Anderson and Corda [4] (1980), Tseng and Lan [5] (1988), McCormick [6] (1989) and Van Dam et al [7] (2001).

The afore-mentioned works in strip theory either worked on a bound vortex circulation correction $\left(\Gamma_{b r}\right)$ or a sectional $\alpha_{s e c}$ correction approach. In the decambering approach

*For correspondence developed by Mukherjee and Gopalarathnam [8] (2006), the camber of the aerofoil is corrected and this change is transferred to both the $\Gamma_{b r}$ and $\alpha_{s e c}$. This work used $C_{m}-\alpha$ along with $C_{L}-\alpha$ data as input. There seems to be a general consensus regarding the presence of multiple solutions, especially at negative $C_{L}-\alpha$ slopes. The presence of asymmetry is more debated, with most of the later works reporting no asymmetric solutions. Paul et al [9] use a UVLM corrected with decambering to study flight dynamics, even for stalled wings in an unsteady condition.

The current study aims to couple a UVLM and a decambering methodology and further understand the nature of the aerodynamic coefficients predicted by the developed model and the variation of solution types with time for the post-stall cases. Furthering the understanding of this methodology will impact applications in flight dynamic simulations, aeroelastic analysis, designing for stall-region operation and pilot training softwares.

\section{Mathematical model}

The wing is simulated by a UVLM coupled with the decambering method [8]. This is used to predict transient aerodynamic coefficients $C_{L}(t)$ and $C_{M}(t)$, along with the wake development.

\section{$2.1 U V L M$}

The UVLM as described by Katz and Plotkin [10] has been implemented. The evolution of the wake at each time step 
is calculated and the influence of the wake on itself and the wing is accounted for.

2.1a Wing definition: In UVLM the wing is represented by a surface that happens to be the camber surface and is divided into $N$ pan $=N X \times N Y$ panels, where $N X$ is the number of panels along the chord and $N Y$ the number of panels along the span. Each panel has a vortex ring and a collocation point. The leading edge of the vortex ring is bound to onefourth of the panel chord length of each panel. The collocation point is placed at three-fourth of the panel chord length of each panel. In figure 1a the panels are grey in colour, the bound vortex rings are blue and the collocation point is the blue dot in each panel.

The no-through flow boundary condition is applied at the collocation point. This implies that the component of velocity, induced by all the bound and wake vortices, normal to the panel and the component of freestream velocity normal to the panel sum up to zero. This is mathematically written in Eq. (1):

$$
\sum_{j=1}^{N p a n}\left(\overrightarrow{v_{b r}} \cdot \hat{n_{i}}\right)_{j}+\sum_{k=1}^{N W}\left(\overrightarrow{v_{w r}} \cdot \hat{n_{i}}\right)_{k}+\overrightarrow{U_{\infty}} \cdot \hat{n_{i}}=0
$$

The unknown quantity in Eq. (1) is $\overrightarrow{v_{b r}}$, which is the velocity induced by the bound vortex rings, and this depends on the circulation strength of the bound vortex ring, $\Gamma_{b r}$. Thus, Eq. (1) is written in a matrix form for each collocation point in Eq. (2):

$$
[I C]_{(N p a n \times N p a n)}\left[\Gamma_{b r}\right]_{(N p a n \times 1)}=-\left[\left(\overrightarrow{U_{\infty}}+\overrightarrow{V_{w r}}\right) \cdot \hat{n}\right]_{(N p a n \times 1)}
$$

where

$$
\overrightarrow{V_{w r}}=\sum_{k=1}^{N W}\left(\overrightarrow{v_{w r}}\right)_{k}
$$

$\overrightarrow{V_{w r}}$ is the sum of velocity induced by all the wake vortex rings at a certain point.

2.1b Wake definition: Eqs. (1) and (2) require that the position and the strength of the wake are known at each time step. The wake behind the wing is characterised by free vortex rings that evolve with time. At each time step the trailing edge vortex rings of the previous time step are shed into the freestream as new wake vortex rings. This is shown in figure 1 . The strength of the wake vortex rings is equal to the strength of the trailing bound vortex rings from the previous time step. Since the wake is characterised by free vortex rings, these rings move at the velocity of the flow field. The velocity at each vertex of the ring is calculated and subsequently the displacement at each time step is calculated using Eq. (3):

$$
\overrightarrow{\Delta x}=\overrightarrow{V_{v}} \cdot \Delta t
$$

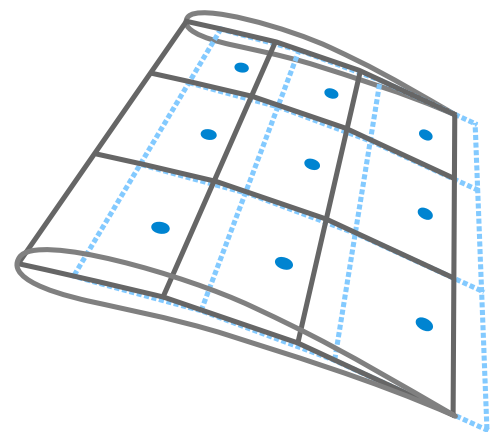

(a) Time step, $\mathrm{n}=0$.

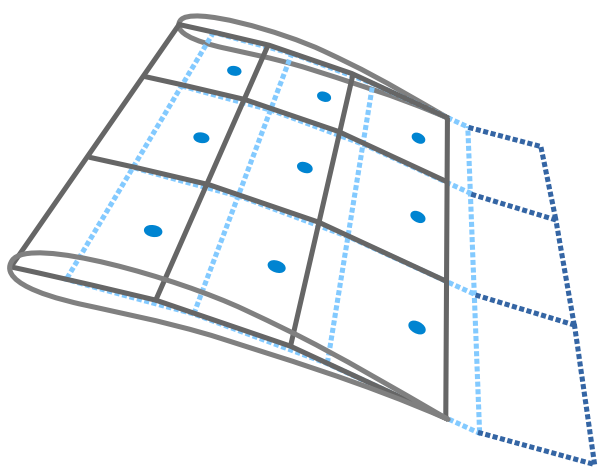

(b) Time step, $\mathrm{n}=1$.

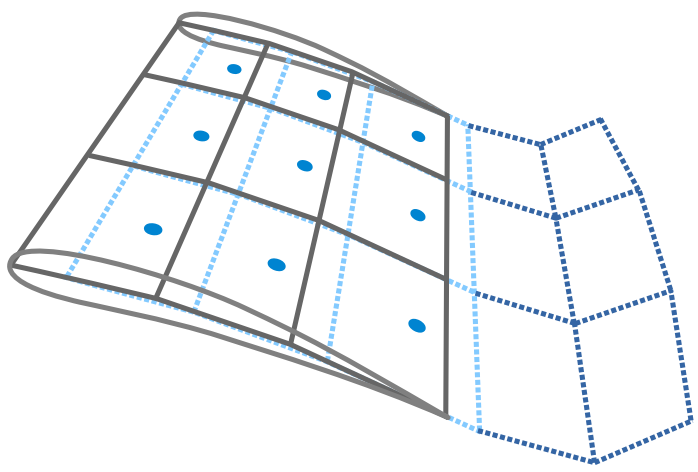

(c) Time step, $\mathrm{n}=2$.

Figure 1. Wake evolution in the UVLM.

where

$$
\overrightarrow{V_{v}}=\overrightarrow{U_{\infty}}+\sum_{j=1}^{N p a n}\left(\overrightarrow{v_{b r}}\right)_{j}+\sum_{k=1}^{N W}\left(\overrightarrow{v_{w r}}\right)_{k}
$$

Thus at each time step the strength and the position of the free wake vortex are known. In order to avoid the singularity in induced velocity that arises when a wake vortex filament comes close to another, a finite Rankine vortex filament core of radius $r_{c}$ and length $l_{c}=l+r_{c}$ is used, $l$ being the length of the vortex filament. 
2.1c Aerodynamic coefficients: The transient coefficient of lift, $C_{L}(t)$, and coefficient of moment, $C_{M}(t)$, are calculated from the bound vortex circulation $\Gamma_{b r}$. The force calculated at each panel has a steady part and an unsteady part. First the pressure difference across each panel is calculated using Eq. (4); then the force is measured using Eq. (5) and from this the lift of the wing is found:

$$
\begin{aligned}
\Delta p_{i, j}=\rho[ & \left(\overrightarrow{U_{\infty}}+\overrightarrow{V_{w r}}\right) \cdot\left(\overrightarrow{\tau_{c}}\right)_{i, j} \frac{\delta}{\delta x} \Gamma_{b r} \\
& \left.+\left(\overrightarrow{U_{\infty}}+\overrightarrow{V_{w r}}\right) \cdot\left(\overrightarrow{\tau_{s}}\right)_{i, j} \frac{\delta}{\delta y} \Gamma_{b r}+\frac{\delta}{\delta t} \Gamma_{b r}\right]
\end{aligned}
$$

where

$$
\begin{aligned}
\frac{\delta}{\delta x} \Gamma_{b r} & =\frac{\Gamma_{i, j}^{n}-\Gamma_{i-1, j}^{n}}{\Delta c_{i, j}}, \\
\frac{\delta}{\delta t} \Gamma_{b r} & =\frac{\Gamma_{i, j}^{n}-\Gamma_{i, j}^{n-1}}{\Delta t}
\end{aligned}
$$

$i$ and $j$ being the panel index in the direction of the panel chord and span, respectively. $\Gamma_{b r}$ is the bound vortex ring circulation. $\Gamma_{i, j}^{n}$ represents the circulation of the bound vortex ring at panel $i, j$ at time step $n$, and the subscript ' $b r$ ' has been omitted for brevity. $\Delta c$ and $\Delta b$ are the average length of the panel in the direction of the chord and span, respectively; $\Delta t$ is the time increment between successive time steps; $\tau_{c}$ and $\tau_{s}$ are the panel unit vector in the direction of the chord and span, respectively; $\rho$ is the density of the fluid used.

The force at each panel of area, $\Delta s=\Delta c \times \Delta b$, is given by Eq. (5):

$$
\Delta \overrightarrow{F_{i, j}}=\left(\Delta p_{i, j} \Delta s_{i, j}\right) \hat{n}_{i, j} .
$$

Let $\overrightarrow{p_{i, j}}=\overrightarrow{U_{\infty}} \times\left(\overrightarrow{\tau_{c}}\right)_{i, j}$. Then the lift of the entire wing is given by Eq. (6). The $C_{L}$ and $C_{M}$ can be calculated from the lift.

$$
\vec{L}=\sum_{i=1}^{N X} \sum_{j=1}^{N Y}\left(\Delta L_{i, j}\right)=\sum_{i=1}^{N X} \sum_{j=1}^{N Y}\left(\Delta \overrightarrow{F_{i, j}} \cdot \frac{\overrightarrow{p_{i, j}}}{\left|\overrightarrow{p_{i, j}}\right|}\right) .
$$

\subsection{The decambering method}

The decambering methodology developed by [8] accounts for the discrepancy between the inviscid aerodynamic coefficients $\left(C_{L}\right)_{p o t}$ and $\left(C_{m}\right)_{p o t}$ and the viscous aerodynamic coefficients $\left(C_{L}\right)_{\text {visc }}$ and $\left(C_{m}\right)_{\text {visc }}$ after flow separation by modifying the effective camber of the lifting line.

2.2a The methodology applied to an aerfooil: The typical flow past an aerofoil at low angles of attack consists of a thin attached boundary layer on the aerofoil surface. The flow follows the camber of the aerofoil as seen in figure $2 \mathrm{a}$.
In the pre-stall regime, the $\left(C_{L}\right)_{p o t}$ and $\left(C_{m}\right)_{p o t}$ predicted using potential flow analysis agree well with both computational and experimental viscous results. With increasing angles of attack, the boundary layer thickens on the upper surface and finally separates as shown in figure $2 \mathrm{~b}$. Here we see that the flow no longer follows the camber of the aerofoil, i.e., the effective camber that the aerofoil enforces on the flow is different from the actual camber. If the change in effective camber can be quantified then the difference $\Delta C_{L}=\left(C_{L}\right)_{\text {visc }}-\left(C_{L}\right)_{\text {pot }}$ and $\Delta C_{m}=\left(C_{m}\right)_{\text {visc }}-$ $\left(C_{m}\right)_{\text {pot }}$ can be found. Conversely, if this difference is known, then the effective camber can be found out.

The change in effective camber is characterised by two parameters $\delta_{1}$ and $\delta_{2}$, which are the flap angles placed on the lifting line of the aerofoil at positions $x_{1}$ and $x_{2}$, respectively. This can be seen in figure 3 . The decambering parameters $\delta_{1}$ and $\delta_{2}$ are related to the difference of the aerodynamic coefficients of the potential flow and the viscous flow regime, $\Delta C_{L}$ and $\Delta C_{m}$, by Eq. (7):

$$
\begin{aligned}
& \delta_{1}=\frac{\left[\Delta C_{L}-2\left(\pi-\theta_{2}\right)+2 \sin \theta_{2}\right] \delta_{2}}{2 \pi} \\
& \delta_{2}=\frac{\Delta C_{m}}{\frac{1}{4} \sin 2 \theta_{2}-\frac{1}{2} \sin \theta_{2}}
\end{aligned}
$$

where

$$
\theta_{2}=\cos ^{-1}\left(1-\frac{2 x_{2}}{c}\right), \quad \frac{x_{2}}{c}=0.8 .
$$

2.2b The methodology applied to an wing $(s)$ : A Newton iteration methodology is used for the $3 \mathrm{D}$ case to find the section-wise decambering. A vortex lattice method is used to predict the potential aerodynamic coefficients $\left(C_{L}\right)_{p o t}$ and $\left(C_{M}\right)_{p o t}$. In order to account for the effect of viscosity on the aerodynamic coefficients, the unit normal vectors at each section are rotated as per the decambering parameters at each section. However the local target angle of attack, $\alpha_{t}$, at each section should be calculated, so that the sectional target aerodynamic coefficients, $\left(C_{L}\right)_{t}$ and $\left(C_{m}\right)_{t}$, can be found. Subsequently the $\delta_{1}$ and $\delta_{2}$ are found such that predicted coefficients $C_{l s}$ and $C_{m s}$ at each section are equal to the target coefficients at the same section.

2.2c Finding local and target angle of attack: If the local lift coefficient $\left(C_{L}\right)_{s e c}$ and the decambering characteristics $\delta_{1}$ and $\delta_{2}$ are known, then the local angle of attack at each section is found using Eq. (8):

$$
\alpha_{s e c}=\frac{\left(C_{L}\right)_{s e c}}{2 \pi}-\delta_{1}-\delta_{2}\left[1-\frac{\theta_{2}}{\pi}+\frac{\sin \theta_{2}}{\pi}\right]+\alpha_{0 l}
$$

where $\alpha_{0 l}$ is the zero lift angle of attack of the aerofoil used.

To find the target alpha at a particular section, the sectional $C_{l s}$ and $\alpha_{s}$ are found. Later the $\delta_{1}$ is perturbed and new values of sectional $C_{l n}$ and $\alpha_{n}$ are generated. The slope 


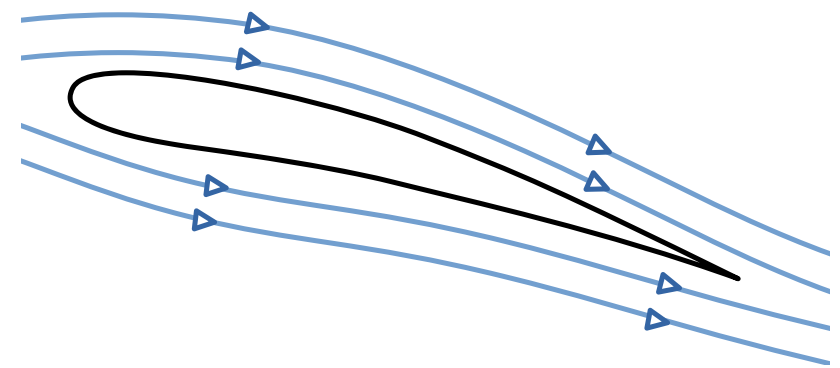

(a) Attached Flow

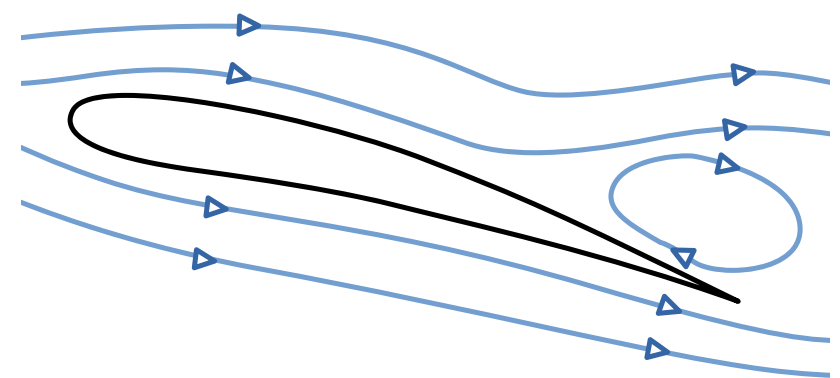

(b) Separated Flow

Figure 2. Flow over an aerofoil.

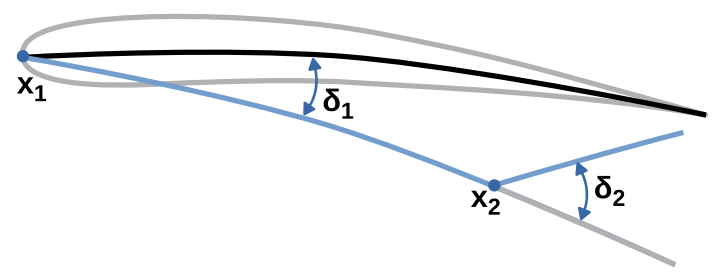

Figure 3. Decambering functions - flap angles.

of the line joining $\alpha_{s}, C_{l s}$ and $\alpha_{n}, C_{l n}$ is now calculated. The point at which this line meets the input data's $C_{L}-\alpha$ curve is noted. This point provides the target angle of attack $\left(\alpha_{t}\right)$ and target coefficient of lift $\left(C_{l t}\right)$ for the particular section.

2.2d Handling multiple solutions: One of the effects of finding targets this way is that multiple solutions are obtained at times depending on the slope of the line. Three instances of the line intersecting the input aerodynamic data are shown in figure 4. Lines L1 and L3 have only one solution. However, this is not the case with line L2, which has three solutions $1-3$.

Selecting a solution out of the three requires more information. In order to aid this selection, a logical switch called lpoststall is used at each section. This switch is TRUE if the section under consideration is stalled. Otherwise, it is FALSE. If there are multiple solutions at a particular section, the status of this switch is checked. If it is a FALSE solution 1 is selected. If it is TRUE, a solution from the multiple post-stall solutions is selected. In order to aid

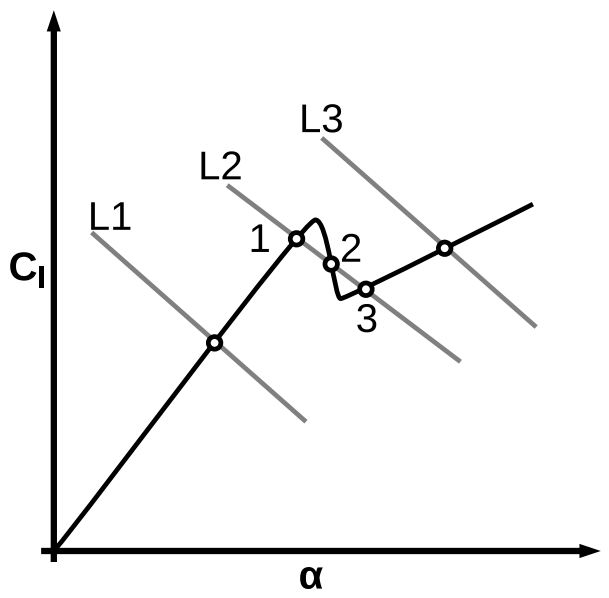

(a) Occurence

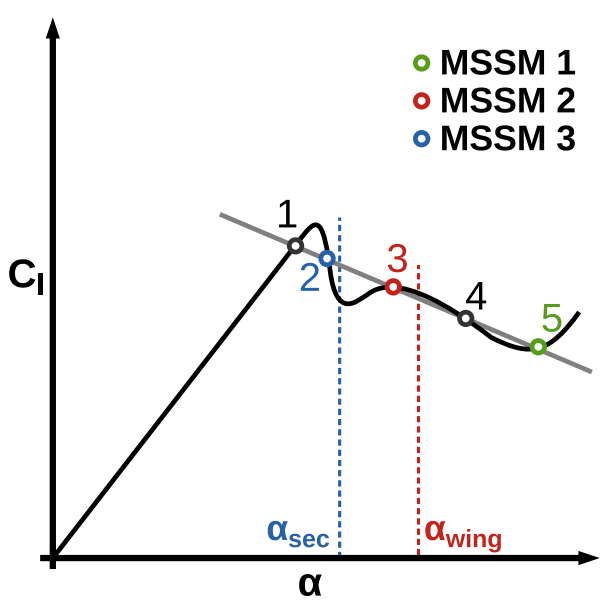

(b) Solution selected for different MSSM

Figure 4. Multiple solutions.

selection between the different post-stall solutions, three Multiple Solution Selection Methods (MSSM) are proposed.

MSSM 1: The final intersection point is selected by this method. The $5^{\text {th }}$ and final solution denoted by the green point in figure $4 \mathrm{~b}$ would be selected as target by this method.

MSSM 2: The intersection point whose $\alpha$ is closest to the wing $\alpha$ is chosen as the target. In figure $4 \mathrm{~b}$ the 3rd solution marked by a red point will be selected.

MSSM 3: The intersection point whose $\alpha$ is closest to the local $\alpha$ calculated from the previous iteration is chosen by this method. Hence the blue point denoting the $2^{\text {nd }}$ solution in figure $4 \mathrm{~b}$ will be chosen.

2.2e Iteration: The steps of the iteration are listed here in brief. 
1. Assume starting $\delta_{1}$ and $\delta_{2}$ values at all sections of the wing.

2. Compute sectional and overall $C_{L}$ and $C_{m}$ of the wing using the Vortex Lattice Method.

3. Compute local effective $A O A, \alpha_{s e c}$, of each decambered section.

4. Obtain target $C_{L}$ and $C_{m}$ from aerofoil data for each cambered section, from the input $2 \mathrm{D}$ data.

5. Calculate residuals $\Delta C_{L}$ and $\Delta C_{m}$ using one of the two schemes.

6. Check if the residuals are within tolerance. If yes, iteration has converged.

7. Else compute Jacobian $J$. Solve $J . \delta x=-F$ to find $\delta x$.

8. Update $\delta_{1}$ and $\delta_{2}$ and continue again from step 2.

\subsection{Coupling decambering and UVLM}

Decambering is a steady method and as such utilises steady $2 \mathrm{D}$ viscous aerodynamic coefficients as input to account for viscous effects. The circulation of the vortex rings, $\Gamma_{b r}$, at each section is modified according to the input $\left(C_{L}\right)_{v i s c}$ and $\left(C_{m}\right)_{v i s c}$. In the UVLM the $\Gamma_{b r}$ at each time step is similarly modified. However while calculating the $C_{l s}$ and $C_{m s}$ from $\Gamma_{b r}$, while iterating for a decambered solution, the unsteady part in Eq. (4) is ignored as we assume each section to be locally steady at that particular time step. Once a converged solution is obtained, the $C_{l s}$ and $C_{m s}$ at each section are recalculated this time taking into account the unsteady part of Eq. (4). This way we obtain 3D unsteady data from 2D steady data.

\section{Validation}

The UVLM code is validated against analytical results, for a suddenly moving elliptic wing, developed by Jones [11], as seen in figure 5. A close match is observed throughout except at $\left(U_{\infty} t\right) / c=0$. This is because the acceleration is infinite in the analytical model at $t=0$. However, numerically only a finite acceleration can be applied.

The wake behind a heaving 3D wing from the current numerical work is compared to the experimental results of a 2D aerofoil (flat plate) in figure 6. The reduced frequency for both is $2.5, \frac{u_{\infty} \Delta t}{c}=0.065$ and $\frac{a}{c}=0.019$, where $a$ is the amplitude of heaving. Three sections along wing-span are considered as follows and as shown in figure 6a: (a) mid-span, shown by the black line when the wing has an aspect ratio $A R=200$, i.e., infinite wing, (b) mid-span, shown by the green line for $A R=7$ and (c) section at $\frac{1}{16} b$, where $b$ is the length of wing-span shown by the red line for $A R=7$. The wake shed from a $2 \mathrm{D}$ aerofoil for similar conditions by Katz and Weihs [12] is shown in figure $6 \mathrm{~b}$.

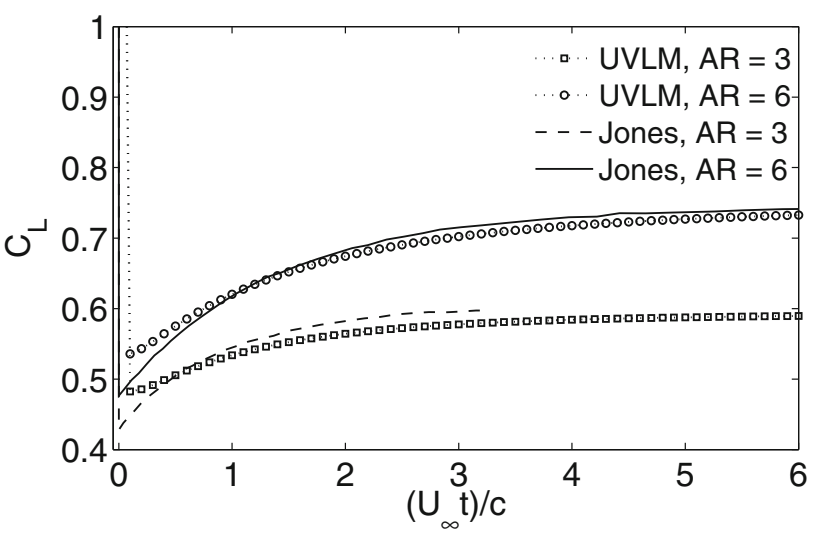

Figure 5. Transient $C_{L}$ of a suddenly moving elliptic wing.

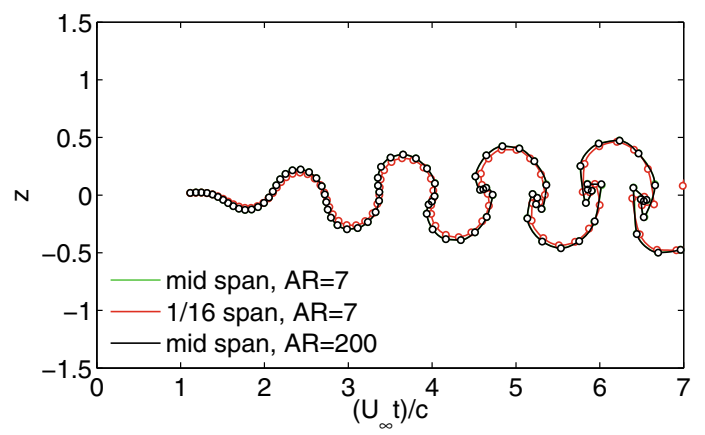

(a) PresentWork

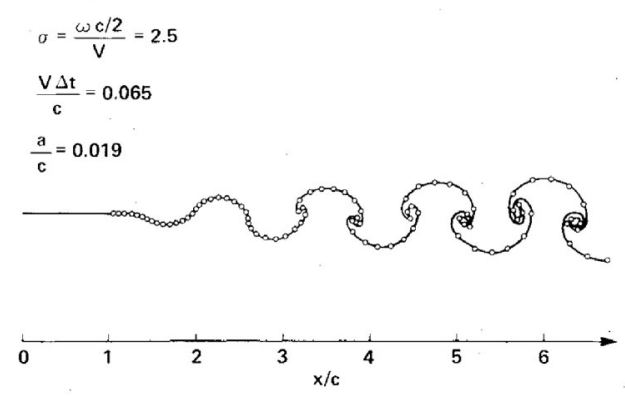

(b) WorkbyKatz[12]

Figure 6. Comparison for heaving flat plate.

The UVLM coupled with decambering is then compared to the UVLM developed by Katz and Plotkin [10] for a plunging wing at three different reduced frequencies, $k=0.1,0.3$ and 0.5 , in figure 7 . An $\alpha$ of $-5^{\circ}$, heaving amplitude of $0.1 c$ and $\mathrm{AR}=4$ are used. The reduced frequency $k$ is given by Eq. (9):

$$
k=\frac{\omega c}{2 U_{\infty}} .
$$




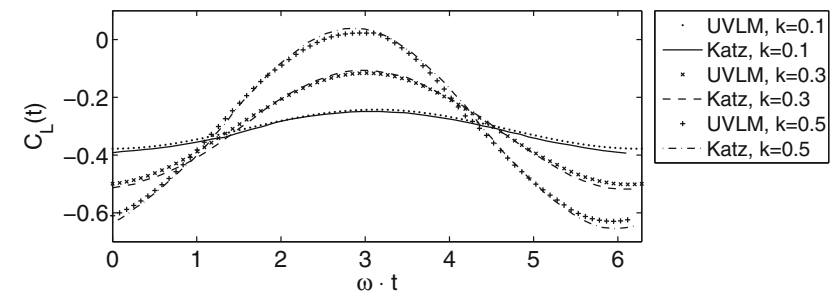

Figure 7. Transient $C_{L}$ of plunging wing.

NACA0009 aerofoil's data at a Reynold's number of $3 \times 10^{6}$, from Abbot et al [13], have been used as input for decambering. The effect of decambering is minimal as the local angles are at all times at pre-stall, and thus we obtain good agreement with the UVLM from Katz and Plotkin [10]. The results from the stand-alone UVLM developed agreed well with the said reference for the same conditions.

\section{Results}

In order to study the stall characteristics of the coupled UVLM and decambering, the case of the suddenly moving wing is looked at in detail. Two aerofoil sections are considered as in the following sections.

\subsection{Rectangular wing: $A R=9$, aerofoil: NACA4412}

The NACA4412 aerofoil data at $R e=3 \times 10^{6}$ from Abbot et al [13] are used as input for this study. The unsteady case is run from $U_{\infty} t / c=0$ to 10 , for angles of attack $-4^{\circ}$ to $20^{\circ}$ for the inviscid case and all three cases of the MSSM. A $\Delta t=0.0625, N X=8$ and $N Y=32$ are used for each case.

4.1a $C_{L}$ variation with $\alpha$ : It is found that at all pre-stall angles of attack $\left(\alpha<13^{\circ}\right)$, of the aerofoil under consideration, a suddenly moving wing attains more than $95 \%$ of the steady $C_{L}$ after moving for $U_{\infty} t / c=7$. Hence the $C_{L}(t)$ from $U_{\infty} t / c=7$ to $U_{\infty} t / c=10$ is averaged at each angle of attack, and this is shown in figure $8 \mathrm{~b}$. The steady $C_{L}$ vs $\alpha$, along with the input NACA4412 aerofoil data, is shown in figure 8a. It is observed that the stall angle of attack of the wing for the steady $C_{L}$, and average of the unsteady $C_{L}(t)$, is $16^{\circ}$. However the trend beyond this point differs significantly for both cases. In figure $8 \mathrm{~b}$ most of the $C_{L}$ values at $\alpha=19^{\circ}$ and $20^{\circ}$ are missing as the decambering failed to converge at certain time steps. It is also observed that not much difference is observed between the MSSM in the steady and unsteady cases for this particular aerofoil. Hence MSSM 1 is used for the further analysis.

4.1b Behaviour of unsteady $C_{L}(t)$ : In order to understand the variation at post-stall in figure $8 \mathrm{a}$ and $\mathrm{b}$, the variation of

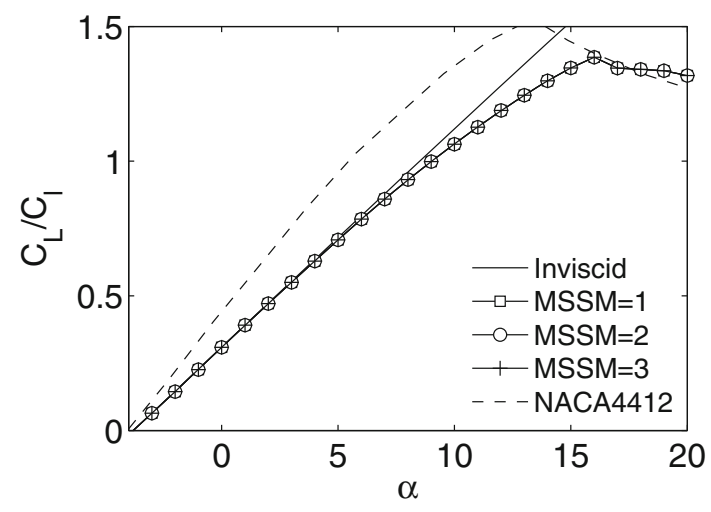

(a) Steady $C_{L}$.

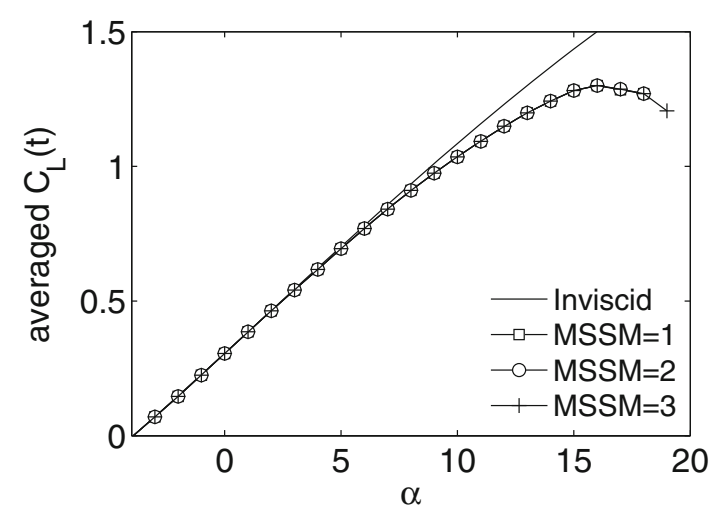

(b) AveragedUnsteady $C_{L}$.

Figure 8. $C_{L}$ vs $\alpha$ for a rectangular NACA4412 wing.

the $C_{L}(t)$ with time is looked at for angles of attack $10^{\circ}, 16^{\circ}$ and $18^{\circ}$ in figure $9 \mathrm{a}, \mathrm{b}$ and c, respectively. Only the inviscid and MSSM 1 cases are looked at for each angle.

It can be seen that for $\alpha=10^{\circ}$ there is a marked difference between the inviscid and MSSM 1 cases. After the initial drop in $C_{L}$ the increase in $C_{L}$ for both the cases is smooth. At $\alpha=16^{\circ}$ the difference between the inviscid and MSSM 1 cases is even more prominent as can be seen in figure $9 \mathrm{~b}$. However, the gain in $C_{L}$ after the initial drop is not smooth as seen in the earlier case. There is a jump in the $C_{L}$ just after $U_{\infty} t / c=9$. The $C_{L}$ solutions beyond this jump attain a lower value than before.

For the $\alpha=18^{\circ}$ run and the MSSM case, the $C_{L}$ loses its smoothness around $U_{\infty} t / c=3$. It is seen that there are blank spaces between certain runs. They represent the time steps at which the decambering has not converged. We require information from the previous time step to calculate the $C_{L}(t)$. If the solution at a previous time step has not converged, the algorithm searches further back in time to find the last converged time step and uses the required information from that time step. The $\Delta t$ is also changed accordingly. The reason for the non-convergence at certain time steps is not known at the moment and needs to be further investigated. 


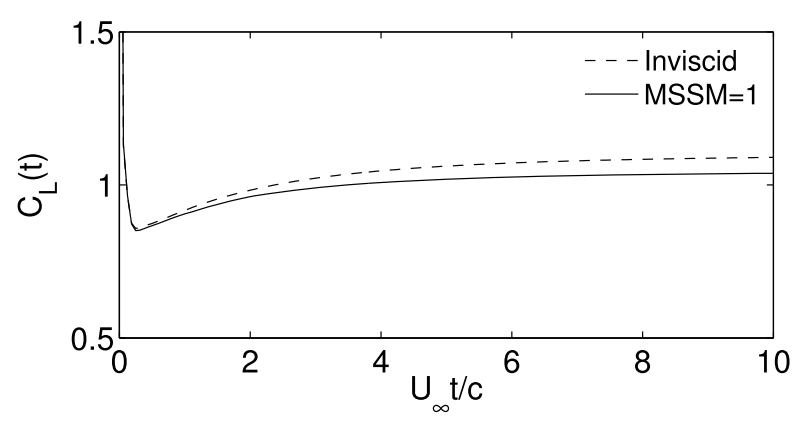

(a) $\alpha=10^{\circ}$.

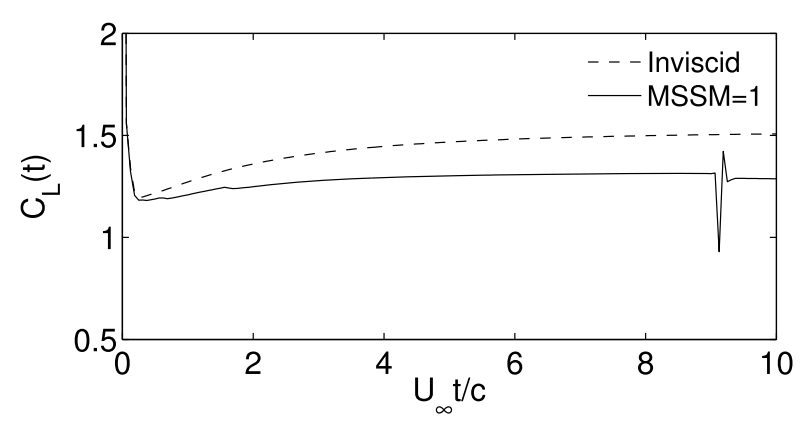

(b) $\alpha=16^{\circ}$.

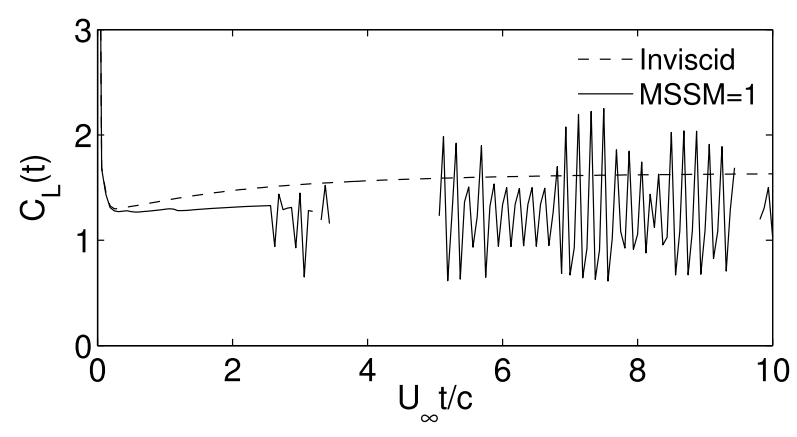

(c) $\alpha=18^{\circ}$.

Figure 9. $C_{L}$ vs $U_{\infty} t / c$ for a rectangular NACA4412 wing.

The transient $C_{L}(t)$ for a series of symmetric wings of different aspect ratios and set into motion with sudden acceleration, from the present work, is compared to that of Katz [14] as shown in figure 10. The time step used is $\frac{U_{\infty} \Delta t}{c}=\frac{1}{16}$ and the angle of attack $\alpha=5^{\circ}$. The wing of infinite aspect ratio represents a $2 \mathrm{D}$ wing or aerofoil.

There are differences between the present results and that of Katz when $\frac{U_{\infty} \Delta t}{c}<0.2$, i.e., just when the motion is beginning. It is also observed that the differences in the initial motion are maximum for the wing of smallest aspect ratio, i.e., maximum $3 \mathrm{D}$ effects and decrease as the aspect ratio increases.

One of the reasons for this can be attributed to the fact that Katz has used 4 chordwise and 13 span-wise panels while the total number of lattices used in the present work

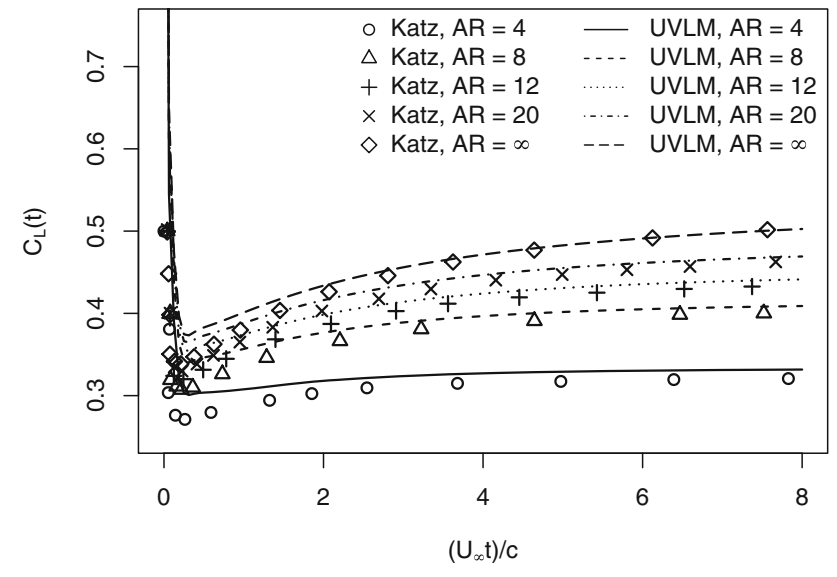

Figure 10. Transient $C_{L}$ of wing started with sudden acceleration.

is $\approx 100$. As a matter of fact, there are no restrictions to this effect using the present code.

4.1c Section $C_{L}(t)$ distribution along span: The span-wise distribution of $C_{L}$ is looked at in this section at different time steps and $\alpha=16^{\circ}$ in order to investigate the reason for these jumps. The variation of the sectional $C_{L}, \alpha$ and $\Gamma$ is shown in figure $11 \mathrm{a}, \mathrm{b}$ and $\mathrm{c}$, respectively.

It is seen that at $t=9.0625$ the $C_{L}$ distribution is symmetric. However, at $t=9.125$ the $16^{\text {th }}$ section from the left sees a huge drop and the nearby sections too see a smaller drop. The $17^{\text {th }}$ section's $C_{L}$ is no longer equal to that of the $16^{\text {th }}$ section and thereby the wing loses its symmetry. The reason for the sudden loss of symmetry is not yet known and has to be explored.

However, after a few time steps there is a tendency to achieve a partial symmetry again. The $C_{L}$ distributions before the jump in $C_{L}$ and at a later time step when the temporal changes have stopped after the jump are shown in figure 12 .

It can be seen that the root sections (the $16^{\text {th }}$ and $17^{\text {th }}$ sections) are stalled at $U_{\infty} t / c=10$. However, this was not the case at $U_{\infty} t / c=9.0625$. The jump in the $C_{L}(t)$ seems to have brought about a stall in the root section, which then became the new steady state. The decambering has jumped from one solution state to another and this results in a lower prediction of $C_{L}(t)$. At higher $\alpha$ s (say $\alpha=18^{\circ}$ ) there is no fixed steady state. The results keeps jumping from one solution to another.

4.1d Wake behind wing: The strength of the wake, i.e. circulation, $\Gamma$ behind a wing at $\alpha=10^{\circ}$ using an inviscid analysis is shown in figure 13a.

When combined with any wake-decay algorithm, this is sufficient to capture the wake structure for viscous flow for small values of $\Delta C_{L}$. The same using decambering is shown in figure $13 \mathrm{~b}$, which primarily shows the effect of decambering in the unsteady flow regime since at $\alpha=10^{\circ}$, there is no flow separation. It is seen that the wake behind the 


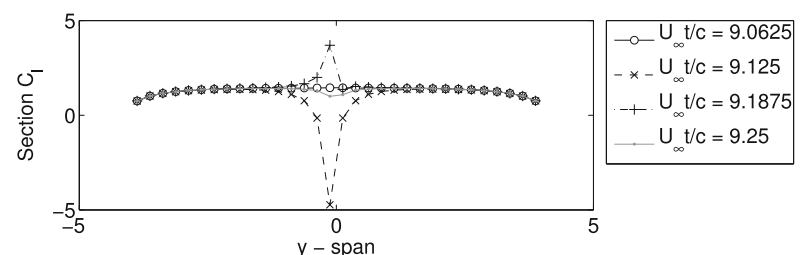

(a) $C_{L}$ vs $y$.

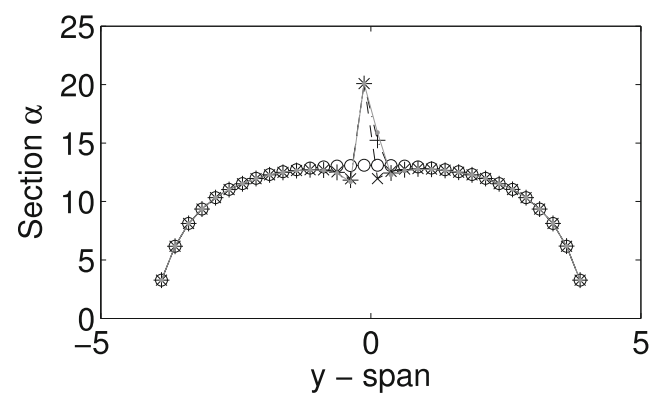

(b) $\alpha$ vs $y$.

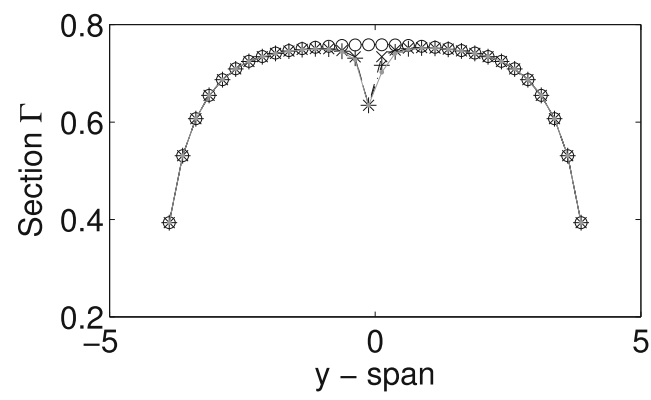

(c) $\Gamma$ vs $y$.

Figure 11. Span-wise variation of different parameters.

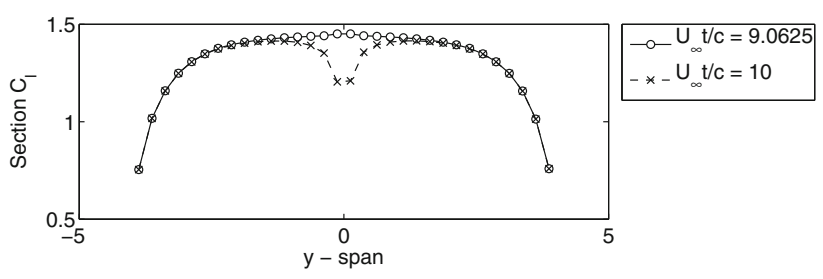

Figure 12. Transient $C_{L}$ of plunging wing.

inviscid wing has wake panels of higher circulation strength compared with the decambered wing. This representation of the wake for a separated flow oversimplifies the wake structure as the shed vortex from the suction surface of the wing is not captured.

\subsection{Rectangular wing: $A R=9$, aerofoil: NACA4409}

For this case, it is seen that considerable difference exists between the decambered and inviscid cases even at pre-stall

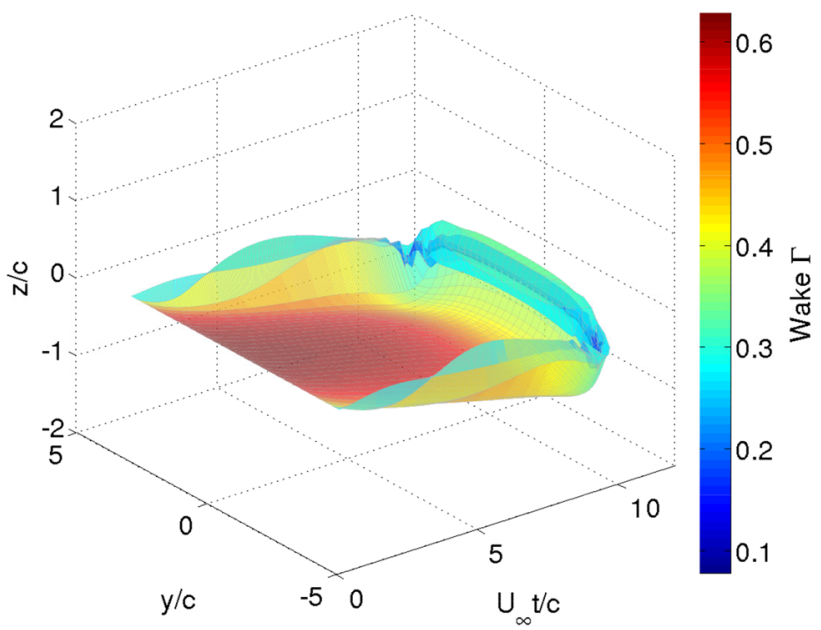

(a) Invicsid.

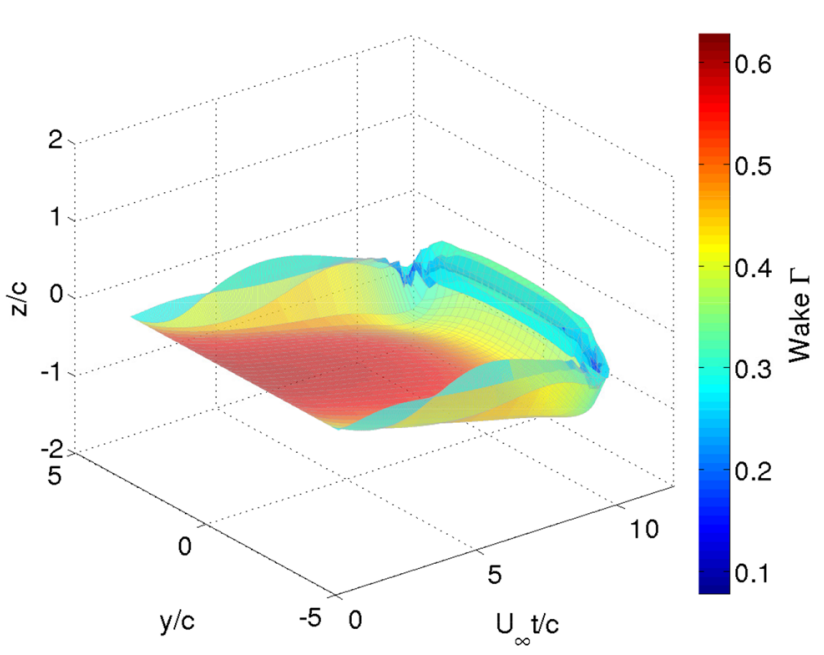

(b) Decambered, $M S S M=1$.

Figure 13. Wake behind a wing at $\alpha=10^{\circ}$.

in the $C_{L}-\alpha$ predicted as shown in figure $14 \mathrm{~b}$ and $\mathrm{c}$ unlike the case shown in figure 8 .

The current methodology is heavily dependent on the 2D aerofoil data used as input. The aerofoil data used in figure 8 are from Abbot while the aerofoil data used for this case are from Naik and Ostowari and these data themselves exhibit the same behaviour as shown in figure 14a. Also, both these aerofoil data are from experiment.

Hence, this behaviour of the current methodology is numerical and physically consistent for the particular case. In other words, the method is valid for the aerofoil data used as input and does not follow the inviscid plot for prestall cases.

It is seen from figure $14 \mathrm{c}$ that in the post-stall region the three MSSM predictions are different. Clearly, this behaviour is numerical in nature since it depends on how these 


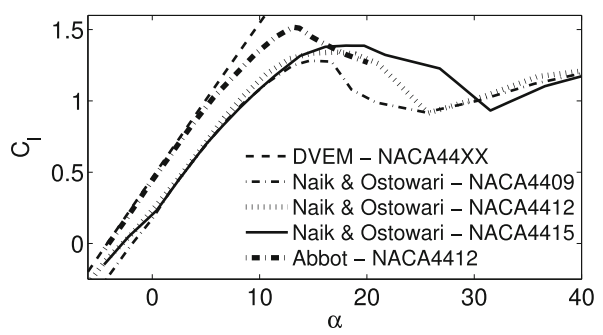

(a) Aerofoil characteristics.

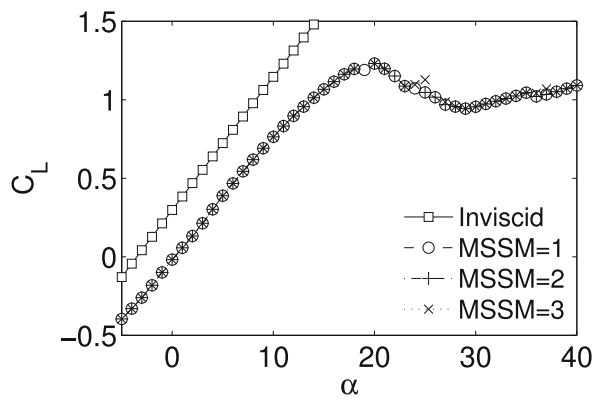

(b) Steady case.

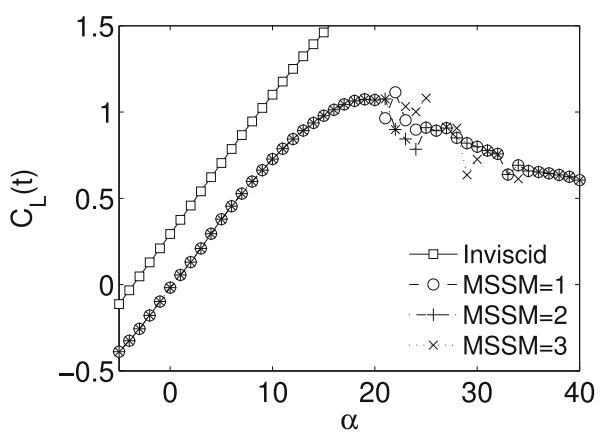

(c) Time-averaged unsteady case.

Figure 14. $C_{L}-\alpha$ for a rectangular NACA4409 wing, $A R=9$.

methods choose a solution from the multiple solutions available at post-stall conditions.

All of these methods lead to converged solutions. It is to be noted here that the final converged solution using either of the MSSMs can be compared to the experiment. However, there is no way to compare the individual solutions chosen during the process of the iteration to the experiment. Hence, if one or more of these finally converged solutions are comparable to the experiment, then it can be considered physically consistent.

More results depicting the numerical behaviour of the MSSMs are shown in figure 15. The prediction from the three methods coincide for $\alpha=20^{\circ}$. At $\alpha=30^{\circ}$, MSSMs 1 and 2 show strong oscillations. This is due to the surge in the unsteady part of the $C_{L}(t)$, i.e., $\frac{\delta}{\delta t} \Gamma_{b r}$ in Eq. (4) when a particular section chooses a different solution. MSSM 3 predicts oscillations of a relatively smaller amplitude but does not converge at all time steps. At $\alpha=40^{\circ}$, a consistent oscillation is observed as the

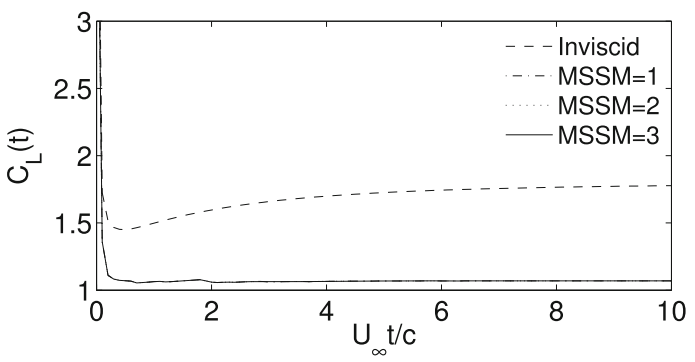

(a) $\alpha=20 \mathrm{deg}$.

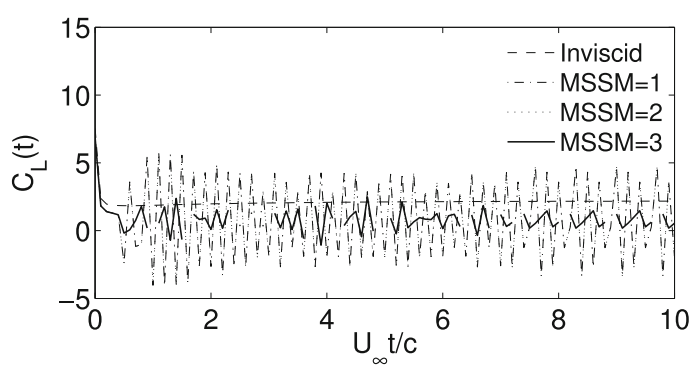

(b) $\alpha=30 \mathrm{deg}$.

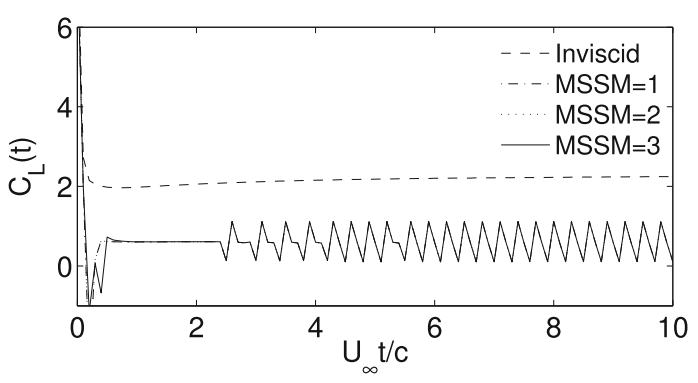

(c) $\alpha=40 \mathrm{deg}$.

Figure 15. $C_{L}(t)$ of a suddenly moving NACA4409 wing, $A R=9$.

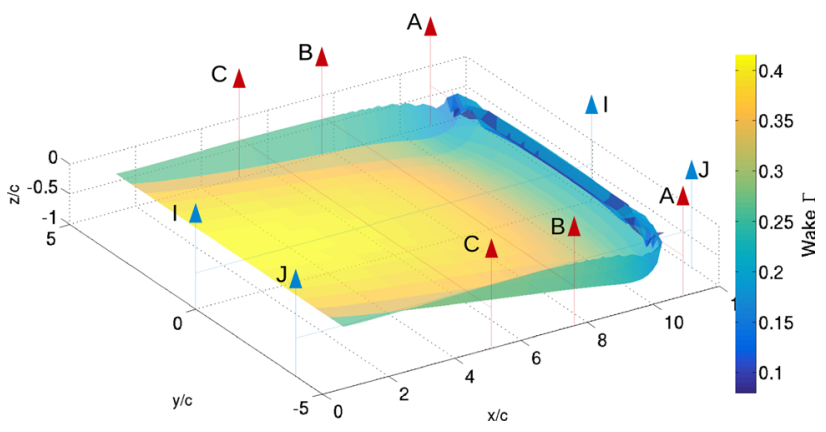

Figure 16. Wake behind a 3D decambered wing.

solution switches from one to the other for convergence and this behaviour cannot be alleviated since there are no multiple solutions available.

The numerical wake profile for a decambered wing using MSSM 1 is shown in figures $16-18$. This is compared with the inviscid case. 


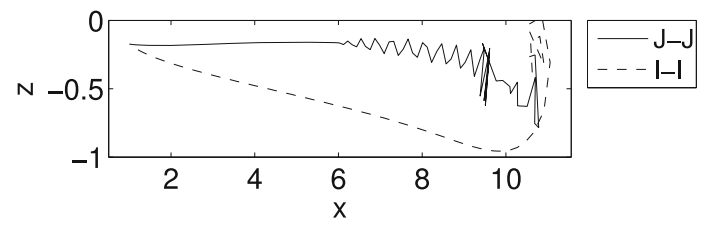

(a) Inviscid.

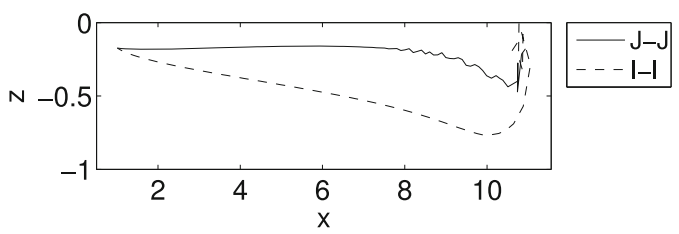

(b) Decambered.

Figure 17. Cross-section of wake at $t=10$, sections along direction of flow.

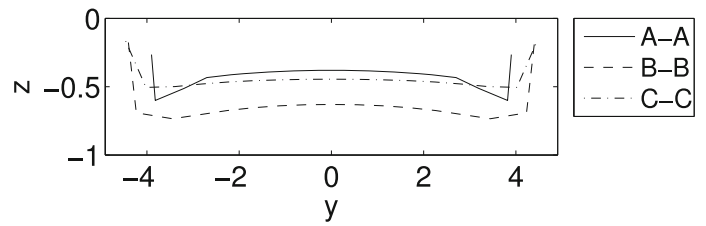

(a) Inviscid

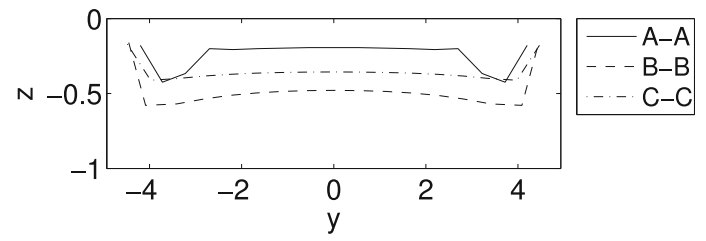

(b) Decambered.

Figure 18. Cross-section of wake at $t=10$, sections along wingspan.

As a final note, not all oscillations in $C_{L}(t)$ can be avoided since either the numerical technique does not encounter the multiple solution zone in the post-stall region or chooses the same solution from the multiple solutions available, which lead to final converged solutions. Hence, while oscillations can be deemed unphysical, the numerical results obtained are converged final solutions. The probable way to alleviate this is to use a root bracketing method instead of the Newton-Raphson method used here.

\section{Conclusion}

A UVLM has been developed, validated and coupled with the decambering methodology in order to make viscous unsteady predictions of aerodynamic coefficients of wings. The time-averaged unsteady $C_{L}(t)$ was compared with the steady $C_{L}$ and it was found that the unsteady $C_{L}$ saw a relative drop at post-stall $\alpha$ s. Different MSSMs did not produce significant variation for the steady and unsteady cases. A study of the transient $C_{L}$ evolution reveals discrete jumps for post-stall $\alpha$ s. These discrete jumps are accompanied by a loss in the $C_{L}$ symmetry along the span but there is a tendency to gain back the symmetry after a few time steps. The jump in the $C_{L}(t)$ is also followed by a move from one solution state to another. As the $\alpha$ increases, the $C_{L}$ jumps' frequency rises and the solution keeps moving to different states with time. Although the wake characterisation technique employed here is sufficient for viscous flows, once the flow separates the current wake characterisation is unable to capture the required details.

\section{Nomenclature}

c chord

$n \quad$ unit normal vector

$A R \quad$ aspect ratio

$I C$ influence coefficient matrix

$J \quad$ Jacobian matrix

$f \quad$ point of separation

$C_{L} \quad$ coefficient of lift

$C_{M} \quad$ coefficient of moment

$C_{n} \quad$ coefficient of normal force

Re Reynolds Number

$\psi \quad$ nascent vortex's streamline angle

$\alpha \quad$ angle of attack

$\Gamma \quad$ circulation

$x, y, z \quad$ spatial coordinates

$\delta x \quad$ residuals

$N_{\text {wing }} \quad$ number of sections

$F \quad$ forcing function

$\mathrm{cr} \quad$ vortex core radius

$N X \quad$ no. of chordwise panels

$N Y \quad$ no. of span-wise panels

Npan total no. of panels

NW no. of wake vortex rings

\section{Subscript}
pot potential flow
visc viscous flow
br bound vortex ring
$w r \quad$ wake vortex ring

\section{References}

[1] Tani I 1934 A simple method of calculating the induced velocity of a monoplane wing. Report of the Aeronautical Research Institute, Tokyo Imperial University 9(3)

[2] Schairer R S 1939 Unsymmetrical lift distributions on a stalled monoplane wing. Master's thesis, California Institute of Technology 
[3] Piszkin S T and Levinsky E S 1976 Nonlinear lifting line theory for predicting stalling instabilities on wings of moderate aspect ratio. Technical report, DTIC Document.

[4] Anderson J D and Corda S 1980 Numerical lifting line theory applied to drooped leading-edge wings below and above stall. J. Aircr. 17(12): 898-904

[5] Tseng J B and Lan C E 1988 Calculation of aerodynamic characteristics of airplane configurations at high angles of attack. Technical Report NASA-CR-4182, Kansas University Center for Research, Inc.; Flight Research Lab.

[6] McCormick B W 1989 An iterative non-linear lifting line model for wings with unsymmetrical stall. Technical report, SAE Technical Paper

[7] Van Dam C P, Vander Kam J C and Paris J K 2001 Designoriented high-lift methodology for general aviation and civil transport aircraft. J. Airc. 38(6): 1076-1084

[8] Mukherjee Rinku and Gopalarathnam A 2006 Poststall prediction of multiple-lifting-surface configurations using a decambering approach. J. Airc. 43(3): 660-668
[9] Paul R, Murua J, and Gopalarathnam A 2014 Unsteady and post-stall aerodynamic modeling for flight dynamics simulation. In: AIAA Atmospheric Flight Mechanics Conference, AIAA SciTech, AIAA 2014-0729

[10] Katz J and Plotkin A 2001 Low-speed aerodynamics. Cambridge Aerospace Series. Cambridge: Cambridge University Press

[11] Jones R T 1940 The unsteady lift of a wing of finite aspect ratio. Technical Report NACA-TN-682, National Advisory Committee for Aeronautics

[12] Katz J and Weihs D 1981 Wake rollup and the kutta condition for airfoils oscillating at high frequency. AIAA $J$. 19:1604-1606

[13] Abbott I H, Von Doenhoff A E and Stivers Jr L 1945 Summary of airfoil data. Technical Report NACA-TR-824, National Advisory Committee for Aeronautics. Langley Aeronautical Laboratory

[14] Katz J 1985 Calculation of the aerodynamic forces on automotive lifting surfaces. J. Fluids Eng. 107: 438-443 